\title{
INSTRUCTIONAL STRATEGY EFFECTS ON THE RETENTION AND TRANSFER OF PROCEDURES OF DIFFERENT DIFFICULTY LEVEL
}

\author{
Otto JELSMA and Jules M. PIETERS * \\ University of Twente, Enschede, The Netherlands
}

Accepted August 1988

In the present study, the effects of two instructional strategies on the retention and transfer of procedures of different difficulty level were investigated. Difficulty level was manipulated by providing a different number of cues during training. The instructional strategies differed with respect to the amount of contextual interference. Sixty-four subjects were randomly assigned to either a high interference group or a low interference group. Retention and transfer were measured immediately following training and after a three-week delay. The dependent variables were number of errors and decision time. Results showed no differences between the two training groups over the various difficulty levels. Results further showed that retention performance increased as fewer cues were available during practice. It is suggested that 'delayed automatization' can account for the observed increment in performance level. It is further suggested that contextual interference may produce delayed automatization of task performance but is only effective if relationships can be discovered in the learning material.

Generalization of a skill over a variety of contexts is an explicit goal of training. It is unlikely that all eventualities can be anticipated and specifically practised (Johnson 1984). Therefore, almost any skill training program not only emphasizes retention of skills that were practised during training, but also their transfer to a variety of different but related task demands in various situations (Annett and Piech 1985; Annett and Sparrow 1985). In an extensive review on psychomotor skill learning, Adams (1987) signalizes a promising new idea to enhance

* We thank Jeroen G.W. Raaijmakers, Richard A. Schmidt, John B. Shea, and Jeroen J.G. van Merriënboer for their useful comments on an earlier version of this article. We also thank Ben Reimerink for implementing the software for the experimental task and Maria Driessen for drawing the Figures.

Requests for reprints should be sent to Otto Jelsma, Department of Education, University of Twente, P.O.B. 217, 7500 AE Enschede, The Netherlands. 
retention and transfer of training, called 'contextual interference'. Shea and Morgan (1979) have borrowed this term from Battig (1979) to describe a learning environment in which mastering of tasks would be slowed down, but subsequent retention and transfer would be enhanced because of increased difficulty in acquiring the tasks. Hence, contextual interference has been experimentally manipulated by having subjects learn a number of tasks in either a random presentation mode (high contextual interference), in which tasks are practised in an unsystematic order across trials, or a blocked presentation mode (low contextual interference), in which all trials on one task are completed before the next task is introduced (e.g., Del Rey et al. 1982; Jelsma and Pieters 1989; Lee and Magill 1983; Shea and Morgan 1979).

Shea and Morgan (1979) performed a prototypical study on the effects of contextual interference on retention and transfer. The task was knocking down six barriers in a prescribed order as quickly as possible. There were several sequences to be learned. The high contextual interference group never practised the same sequence on successive trials while the low contextual interference group completed all trials for one particular sequence before going on to the next one. It was found that practising under conditions of high contextual interference was detrimental to performance during training, but produced better retention and better transfer to new sequences than practising under conditions of low contextual interference.

Shea and Morgan (1979) argued that the unpredictability in the learning situation forced subjects in the high contextual interference group to use multiple processing strategies that resulted in deeper cognitive processing during acquisition. Shea and Zimny (1983) have described a theoretical processing model of contextual interference. They proposed that contextual interference effects result from the concurrent presence of items of more than one task in working memory which facilitates elaborative processing both across tasks (inter-item level) and within a task (intra-item level) in the high contextual interference condition, but facilitates only elaborative processing at the intra-item level in the low contextual interference condition. Lee and Magill (1983) offered a slightly different view that explained the effects of contextual interference primarily in terms of greater cognitive effort. According to Lee and Magill (1983) the unpredictability in the learning situation fo:ced subjects in the high contextual interference group to actively regenerate action plans from trial to trial while a predictable 
sequence of trials permitted subjects in the low contextual interference group to rely on the action plan generated for the previous trial. Whatever explanation may be true, a growing body of evidence is emerging that suggests that randomly ordering practice trials may produce less accurate training performances but facilitates retention and transfer (Del Rey et al. 1982; Jelsma and Pieters 1989; Lee and Magill 1983, 1985; Lee et al. 1985; Salmoni et al. 1984; Shea and Morgan 1979; Shea and Zimny 1983, 1988).

Despite different explanations for the contextual interference effects, there seems to be agreement over the fact that conscious mechanisms subserving the translation from intention-to-action (controlled processes) play an important role in the production of the observed contextual interference effects. Also, Fisk and Schneider (1984) have presented data which strongly suggest that learning and controlled processing are closely linked. They maintain that for learning to take place, controlled processes should be utilized by the learner. Schneider and Shiffrin's (1977; Shiffrin and Schneider 1977) dual processing theory distinguishes between controlled processes and automatic processes to indicate two qualitative different forms of human information processing. Controlled processes are slow, require effort, are easy to modify, but place severe limits on working memory capacity. Automatic processes are fast, occur with little or no effort, are not limited by working memory capacity, but are difficult to modify once developed. Some functions of controlled processes are to develop new automatic processes, to deal with novel situations, to maintain information in working memory, to activate automatic processes, and to inhibit or modify existing automatic processes. Following Schneider and Shiffrin, the amount of controlled processing during the course of learning is expected to determine long term memory storage.

Recently, Shea and Zimny (1988) have interpreted the contextual interference effects in terms of the controlled/automatic processing dichotomy. They suggest that automatic processing is facilitated in the low contextual interference condition because no decisions among tasks are necessary, and configural knowledge of the tasks is sufficient to guide good performance. This configural knowledge is not sufficient, however, for recall which necessitates more precise, detailed knowledge to distinguish among the tasks. Thus, learning under a blocked practice schedule means that subjects do not have to attend to as many aspects of the task on each trial as they would if they were given a different 
task on each trial. So, they do not encode aspects of the tasks which would help in differentiating one task from another. This means that there is less information available when the subjects are asked to recall and they perform less well. The high contextual interference condition facilitates more active, controlled processing, and this processing results in a memorial representation that is adequate for retrieval in retention and transfer.

Following Shea and Zimny's (1988) suggestion that contextual interference is having an effect on how much of the input is actively processed, it can be argued that the differences in performance on retention and transfer tests after practising under conditions of low and high contextual interference can be accounted to the intensity of controlled processing that the conditions call for. It should be noted here that the goal of studies on contextual interference effects is to attain mastery level and not the subsequent development of automaticity, which in general requires hundreds or even thousands of trials. It is suggested that encouraging the use of controlled processes in the initial stages of skill acquisition can enhance subsequent retention and transfer performance, although this is likely to slow down the speed of acquisition. This intriguing notion seems to conflict with the general purpose of many training programs that skills should be rapidly acquired, that is, controlled processing should be quickly reduced or task performance should be quickly automatized (e.g., Eberts 1987). However, it is in line with research on the contextual interference effect that suggests that fast improvements in performance level obtained in highly predictable learning environments - possibly leading to an accelerated automatization of task performance - should be sacrificed if retention and transfer is the primary goal of training. Johnson (1984) has argued that predictability in training is definitely considered a key element in automatization of task performance. Decreasing predictability in the learning situation - for instance by means of contextual interference - could foster controlled processing and consequently delay automatization of task performance.

Indeed, results of studies on the contextual interference effect are very impressive. However, by our knowledge all of these studies used relatively easy tasks. If more complicated tasks have to be learned, effects of contextual interference on retention and transfer are not clear yet. Then, learning under conditions of high contextual interference may result in a too difficult learning situation, possibly causing the 
positive effect of contextual interference on retention and transfer to fade away. Therefore, before application of contextual interference in instructional settings can be recommended, its relation with task difficulty should be investigated. In addition, it is hardly known if, as Shea and Zimny's (1983) 'elaboration' point of view suggests, a particular overlap between the tasks practised, is a prerequisite to produce the contextual interference effect. That is, it is unknown if the procedures through which the tasks' goals are achieved must have 'elements' in common, for instance in terms of a number of corresponding procedural steps (see also, Kieras and Polson 1985), in order to establish under conditions of high contextual interference a memorial representation more adequate for retention and transfer. We know of only one study that deliberately used unrelated tasks (with no procedural steps in common) to demonstrate the contextual interference effect (Magill and Lee 1984) and thus to find support for Lee and Magill's (1983) 'cognitive effort' explanation for contextual interference effects. The effect was found indeed, which would provide support for the 'cognitive effort' rather than the elaboration' explanation. However, still the tasks used were relatively easy and so far it is unclear if their results can be generalized to more complicated tasks.

The purpose of the present study is to determine the effect of contextual interference on the retention and transfer of procedures of different difficulty levels that have no procedural steps in common. A procedure is conceived of as a series of different overt or covert actions that must be performed in a sequential or steplike fashion in order to achieve the task goal (e.g., Romiszowski 1984). Difficulty level is varied by providing different amounts of cues concerning the procedures to be learned. A cue is defined as a perceptual event that is used to enhance important characteristics of a display. According to Lintern (1985), providing more cues can reduce the difficulty level of the task in that the task is easier to perform.

Based on results of earlier studies on the contextual interference effect we predict that training under conditions of high contextual interference will lead to a low performance level during training, but to a high performance level during retention and transfer. In addition, we predict an interaction between the amount of contextual interference and difficulty level on retention tests and transfer tests. For low difficulty levels, that is, if many cues were available during training, practice under conditions of high contextual interference will enhance 
retention and transfer performances because the use of controlled processes is intensified. For high difficulty levels, that is if few cues were available during training, practice under conditions of high contextual interference will have a negative effect on retention and transfer performances because the learning situation has become too difficult. Finally, as for instance Lintern (1985) and Eberts (1987) have argued, the provision of cues during training may have beneficial effects on actual task performance. A strong drawback is that subjects often form a dependency on the cues which affects their performance negatively if the cues are withdrawn later on. Therefore, we predict that the time necessary to acquire the procedures will be short if many cues are available and long if few cues are available. Acquisition time being fixed more cues will lead to a higher performance level during training. However, performance level on retention tests will decrease as more cues were provided during training. If the number of cues of the practised procedures and of variants of these procedures tested on transfer is held constant, performance level on transfer tests will increase as more cues are available.

\section{Method}

\section{Subjects}

Sixty-four right handed university students $(21$ males and 43 females; mean age $=$ 20.8 years, $S D=2.3$ years) participated in the experiment for course credit.

\section{Apparatus}

The experiment was conducted in a sound proof cubicle and was controlled by a PDP-11/03 mini computer. Subjects were seated at a table in a comfortable chair. A monochrome screen was placed at eye-level in front of the subject. Within easy reach, opposite the midline of the subject's body, a control panel with four soft touch direction keys was mounted on the table. The keys were used to specify the procedures to-be-learned. Errors and decision times (time between the onset of an auditory starting-signal and initiation of cursor displacement) were recorded and stored on 5.25" floppy discs for later analyses. Auditory feedback was realized as follows: Whenever the subject hit a wrong key a signal was channelled to an audio amplifier and fed back to the subject via a loudspeaker. Like decision times, the number of errors was registered and stored on the 5.25" floppy discs. 


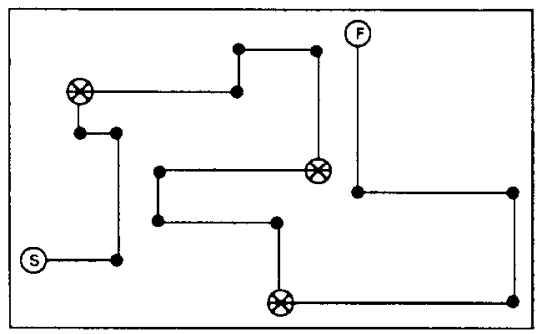

Fig. 1. A track.

Task

In modern society, the importance of tasks, in which information of (computer)screens has to be interpreted in order to produce particular action sequences (for instance the deletion of a character in a text-editing program), is increasing. For that reason, we have designed the following computer-based task to conduct our study. Subjects had to learn to perform four different procedures by making use of particular screen information and the specially constructed control panel. They had to direct a cursor along four different tracks as quickly as possible and with minimal errors. Each track required a different procedure. A rectangular framework bordered the area in which a track had to be followed (fig. 1).

Each track was made up of 16 straight lines and 15 right angles. The fourth, eight and twelfth angular point were marked by an asterisk. These angular points are referred to as action points. During task performance the lines were not visible. The total length of the lines was $60 \mathrm{~cm}$ for each track. Both horizontally and vertically, there were never more than two angular points in a direct line. For each track, corresponding angular points had different coordinates. Also the positions of starting point and finish indicated by the characters ' $S$ ' and ' $F$ ', respectively, were for each track unique. so, perceptually the four tracks showed little resemblance.

As can be seen from table 1 , the procedures through which the tracks could be followed were unique as well. That is, the procedures had no procedural steps in

Table 1

The procedures through which the four tracks could be followed. (R: Right, L: Left, U: up, D: Down.)

\begin{tabular}{lllll}
\hline Procedure & \multicolumn{2}{l}{ Procedural steps } & & IV \\
\cline { 2 - 5 } & I & II & II & R-U-L-U \\
\hline I & R-U-L-U & R-U-R-D & L-D-R-D & R-D-L-D \\
II & R-U-I -D & L-U-L-U & R-D-R-U & D-R-U-L \\
III & D-L-U-L & D-R-U-L & U-R-D-R & L-D-L-D \\
IV & L-U-R-U & L-U-L-D & L-D-R-D & \\
\hline
\end{tabular}


common and thus there was no overlap between the four procedures. A procedural step was conceptualized as a response combination of four discrete actions on the control panel.

To set the cursor going, subjects had to perform at each action point (including the starting point ' $S$ ') the right procedural step by entering the right response combination on the control panel. So, a procedural step specified the route for the cursor to the next action point. The cursor moved from one action point to another only if the right procedural step was performed, that is, only if the right response combination was entered. It's speed was $12 \mathrm{~cm} / \mathrm{sec}$. For instance, in order to direct the cursor in fig. 1 (see table 1, procedure I) from the first action point (starting point ' $S$ ') to the second (fourth angular point) the response combination Right, Up, Left, Up should be entered on the control panel. To indicate that a next procedural step could be performed, an auditory signal was rendered the moment the cursor arrived at the adjacent action point. Auditory feedback was provided as soon as the subject pushed in a wrong direction key. In that case, an error was registered. At the same time the part of the procedural step that had been entered correctly was displayed in the bottom left corner of the screen by means of the characters ' $R$ ' (right), 'L' (left), 'D' (down) and ' $U$ ' (up). Subjects just had to complete the procedural step to proceed.

Cues were used to create four levels of task difficulty. The levels 1 through 4 were created by marking with a dot $12,8,4$ and 0 of the intermediate angular points,

A

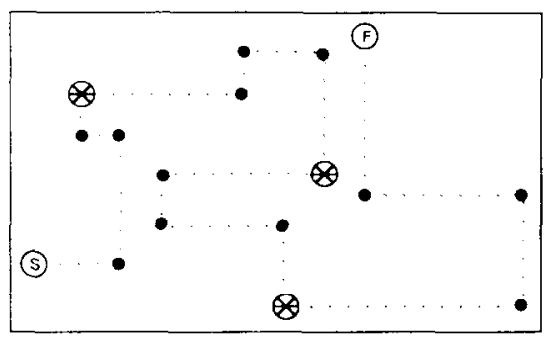

$\mathrm{C}$

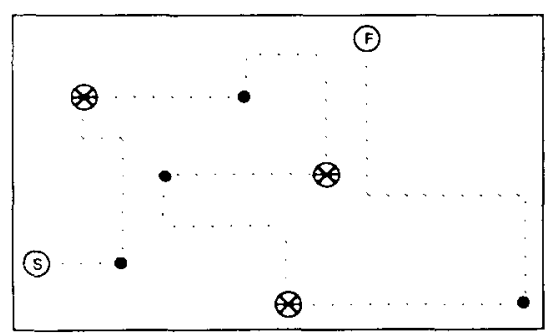

B

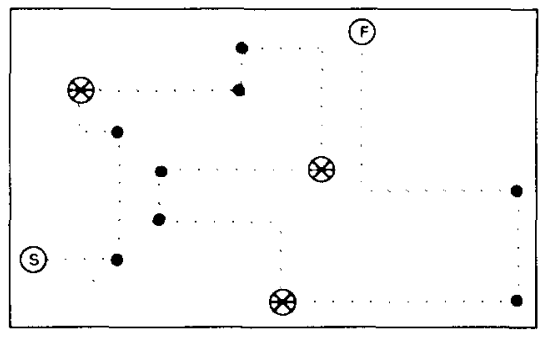

D

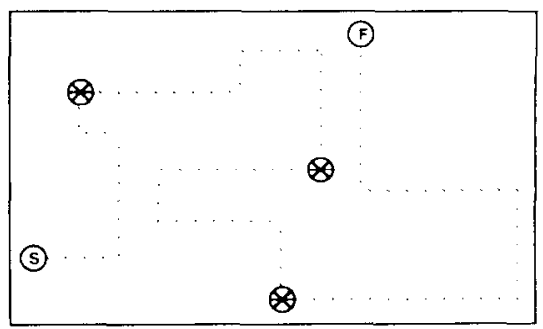

Fig. 2. The four difficulty levels of one of the procedures. (A level 1; B level 2; C level 3; D level 4. The dotted lines were not visible for subjects.) 
respectively. Thus, at level 1 all intermediate angular points were marked (fig. 2A). At level 2 always the first two angular points after an action point were marked (angular points $1,2,5,6,9,10,13,14$, fig. 2B). At level 3 only the first angular point after an action point was marked (angular point 1, 5, 9, 13, fig. 2C). And at level 4 no intermediate angular points were marked (fig. 2D).

\section{Procedure}

The experiment was divided into two sessions. The first session consisted of a training. a retention and a transfer test. The second session was conducted after a three weeks delay and consisted of a retention and transfer test only. At the beginning of each session subjects were given standardized written instructions to explain the purpose of the task. It was also explained that the experiment was completely computer controlled. Training, retention and transfer tests are described separately.

\section{Training}

The 21 males and 43 females were randomly assigned to either a high contextual interference group (HICON, $N=32$ ) or a low contextual interference group (LOCON, $N=32$ ). Each subject performed 40 practice trials, 10 trials for each procedure. Subjects in the LOCON group always completed all trials for a procedure of one particular difficulty level before a new procedure of another difficulty level was introduced. The design was completely counterbalanced for difficulty level and order of presentation of the procedures. Subjects in the HICON group practised the four procedures of different difficulty levels in a random order. The same procedure was never practised twice in succession.

Prior to the start of the training subjects got acquainted with the way the cursor was controlled by practising at a fake track during three minutes. Its structure was completely visible, so only the cursor control was practised. Next a message on the screen stated that the experiment started in three seconds. Prior to each trial the structure of the track was displayed completely during three seconds. After that, the straight lines of the track disappeared. After another second an auditory signal indicated the beginning of a trial. Subjects now had to infer the right procedure from the starting point ('S'), finish (' $F$ '), from the action points marked by an asterisk and, from a number of marked intermediate angular points $(0,4,8$, or 12$)$. To indicate that a new response combination had to be entered on the control panel the auditory signal was repeated the moment the cursor arrived at the next action point. A trial was completed as soon as the cursor reached the finish. After completing a trial subjects obtained knowledge of results (KR), that consisted of the total number of errors they made and of the time it took to complete the trial. KR was displayed four seconds on the screen. Following KR presentation a message on the screen informed subject that the next trial started in three seconds. For each trial total number of errors and total decision time were recorded. Decision time was operationalized as the time between onset of the auditory signal at an action point and initiation of cursor displacement. Total decision time represented the sum of the four separate decision times. An error was made as soon as subjects hit a wrong direction key. As soon as 40 practice trials 
were performed a message on the screen reported the end of the training. Following training a short break of 10 minutes was provided.

\section{Retention}

Both training groups performed 12 retention trials, three for each track. Subjects now had to infer the complete procedure only from the starting point ' $S$ ', the three intermediate action points and the finish ' $F$ ' that were displayed on the screen and that were specific for each track. Prior to a trial, the structure of the tracks was not displayed on the screen. Furthermore, no intermediate angular points were displayed, so all procedures were assigned the highest difficulty level. In order to control for individual differences in self-regulating behavior (Simons and Beukhof 1987), procedures for feedback presentation were identical to procedures in training. Retention trials were performed in a blocked order. For each trial total number of errors and total decision time were recorded.

Following the first retention test subjects were given a two minute rest. After that the first transfer test was administered. After a three-week delay a second retention test identical to the first and a second transfer test was administered.

\section{Transfer}

To assess transfer performances subjects had to direct the cursor along mirror images of the original tracks. For each subject, mirrored tracks had the same difficulty level as in their original forms. Both training groups performed 12 transfer trials, three for each mirrored track. At the first test the original tracks were mirrored against the horizontal axis and at the second test against the vertical axis. After a three weeks delay the second transfer test was administered following the second retention test. Procedures for feedback presentation were again identical to procedures in training. As in training, prior to each trial the complete structure of a track was displayed on the screen. Transfer trials were performed in a blocked order. For each trial total number of errors and total decision time were recorded.

\section{Analysis}

Statistical analyses were performed separately on training, retention and transfer data. MANOVAs on errors and decision times and univariate ANOVA tests for each dependent variable were conducted. Level for achievement of statistical significance was set at 0.05 . All three-way interactions were ignored. MANOVA and ANOVA analyses over Condition (HICON vs. LOCON), Difficulty level (1 to 4) and Trials ( 1 to 10) with repeated measures on the latter factor were carried out to test for significance of training effects. Furthermore, MANOVA and ANOVA analyses over Condition (HICON vs. LOCON), Difficulty level (1 to 4 ) and Testing moment (immediately following training vs. 3 weeks delay) with repeated measures on the latter factor were conducted to test for significance of both retention and transfer effects. 


\section{Results and Discussion}

\section{Training data}

The MANOVA revealed a significant effect for Condition, $F(2,60)=6.74, p<0.01$. For the HICON group the mean number of errors (MNE) was 1.58 and for the LOCON group 1.14. The main decision times (MDT) were $21.95 \mathrm{sec}$. and $17.06 \mathrm{sec}$, respectively. ANOVAs on both errors, $F(1,61)=5.01, p<0.05$ and decision times, $F(1,61)=12.71, p<0.01$ agreed with the multivariate results. Thus, training data clearly support our hypothesis that training under conditions of high contextual interference leads to a lower performance level as compared to training under conditions of low contextual interference. This result is consistent with earlier research on the contextual interference effect.

The MANOVA further indicated a significant effect for Difficulty level, $F(6,372)$ $=12.50, p<0.01$. MNE over the four difficulty levels was consecutively 0.82 (level 1 ), 1.02 (level 2), 1.60 (level 3) and 2.00 (level 4). MDT was $16.01 \mathrm{sec}$ (level 1), $18.51 \mathrm{sec}$ (level 2), $22.30 \mathrm{sec}$ (level 3) and $21.20 \mathrm{sec}$ (level 4). The ANOVAs on error data and on decision time data revealed a significant effect for Difficulty level as well, $F(3,186)=$ 18.26, $p<0.01$ and $F(3,186)=15.76, p<0.01$, respectively. Thus, training data support our hypothesis that the provision of more cues results in a higher performance level. As Lintern (1985) has suggested, this result can be accounted to a reduction in task difficulty.

The MANOVA also indicated a significant Trial effect, $F(18,1116)=43.00, p<$ 0.001 . Observations of training data showed a typical curvilinear training performance curve for both errors and decision times. For both dependent variables, the ANOVAs indicated a significant Trial effect as well, $F(9,558)=103.91, p<0.001$ and $F(9,558)$ $=242.41, p<0.001$, respectively. The performance curve for the LOCON group declined more rapidly than the performance curve for the HICON group. At the end of training, both practice groups achieved a similar performance level. The MANOVA showed a significant Condition $\times$ Trial interaction effect, $F(18,1116)=2.05, p<0.01$. Likewise, the subsequent ANOVAs on both error data and on decision time data revealed a significant Condition $\times$ Trial interaction effect, $F(9,558)=2.76, p<0.01$ and $F(9,558)=2.82, p<0.01$, respectively. Given that the difference between the two practice groups lied in the initial stage of training, it is argued that it was more difficult for the high contextual interference group than for the low contextual interference group to master the procedures. Again, this result is consistent with earlier research on the contextual interference effect.

Finally, the MANOVA indicated a significant interaction of Difficulty level with Trial, $F(54,3348)=7.30, p<0.01$. ANOVAs on both error data, $F(27,1674)=7.98$, $p<0.01$ and decision time data, $F(27,1674)=7.90, p<0.01$ agreed with the multivariate result. The higher difficulty levels resulted in a lower performance level during the first period of training (about the first six trials), but at the end of training performance curves for all four difficulty levels converged in a similar performance level which remained stable over the latter training trials. Thus, eventually the procedures were mastered at all four difficulty levels. 


\section{Retention data}

Fig. 3 presents the group's retention performance over the four difficulty levels for MNE (fig. 3A) and for MDT (fig. 3B).

Training data clearly have shown that it was more difficult for the high contextual interference group to master the procedures. In our view, this means that this group made a more intensive use of controlled processes during practice, which was expected to facilitate retention performance. However, the MANOVA and subsequent ANOVAs on retention data revealed no significant Condition effects. Therefore, with respect to retention data, the results of our study do not support earlier studies on the contextual interference effect.

Also contrary to prediction, no significant interaction was found between condition and difficulty level. Over all four difficulty levels the small advantage of the HICON group over the LOCON group was about the same. Thus, we did not find support for our hypothesis that, as compared to subjects in the LOCON group, subjects in the HICON group would benefit from their intensified use of controlled processes in practising relatively easy tasks, but that this would be detrimental in practising more complicated tasks because of a too difficult learning situation.

The MANOVA did indicate a significant effect for Difficulty level, $F(6,372)=372$. $p<0.01$. MDT over the four difficulty levels was $62.32 \mathrm{sec}$ (level 1), $59.14 \mathrm{sec}$ (level 2), $54.67 \mathrm{sec}$ (level 3), and $50.25 \mathrm{sec}$ (level 4), respectively. The ANOVA on decision time data reached statistical significance as well, $F(3,186)=6.30, p<0.01$. This result suggests that procedures of higher difficulty levels were remembered best. As training

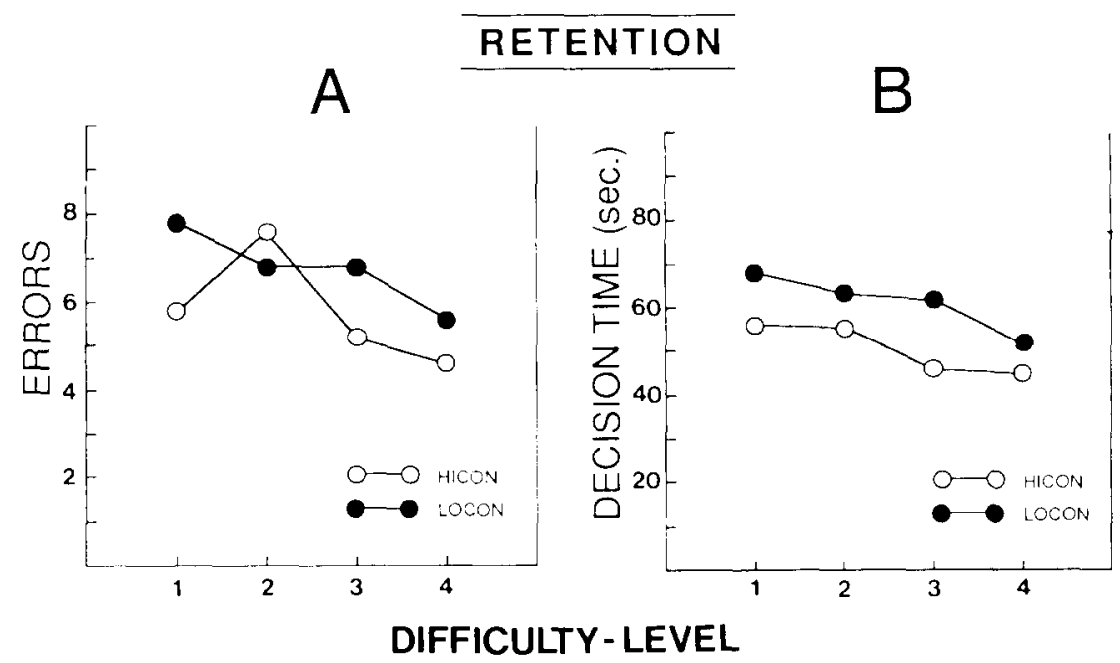

Fig. 3. The mean number of errors (4A) and the mean decision times (4B) on retention tests of the high contextual interference group (HICON) and the low contextual interference group (LOCON) as a function of the difficulty level of the practised procedures. 
data have shown, it was more difficult to master those procedures. Thus, it can be argued that during training performance for procedures of higher difficulty levels depended more heavily on controlled processes than performance for procedures of lower difficulty levels. This result supports our general hypothesis that intensifying the use of controlled processes or delaying automatization of task performance during training could be the underlying mechanism that facilitates learning.

The MANOVA further indicated a significant effect for Testing moment, $F(2,61)$ $=8.02, p<0.01$. The subsequent ANOVA on error data reached statistical significance as well, $F(1,62)=14.89, p<0.01$, indicating the expected performance decrement between the first $(\mathrm{MNE}=5.52)$ and second test $(\mathrm{MNE}=6.97)$.

\section{Transfer data}

Fig. 4 presents the group's transfer performance over the four difficulty levels for MNE (fig. 4A) and for MDT (fig. 4B).

Like on retention data, the MANOVA and subsequent ANOVAs on transfer data revealed no significant Condition effects. Thus, also with respect to transfer data, the results of our study do not support earlier studies on the contextual interference effect. In addition, the MANOVA and ANOVAs revealed no significant interaction between condition and difficulty level. Like retention data, over all four difficulty levels the small advantage of the HICON group over the LOCON group was about the same.

The MANOVA indicated a significant effect for Difficulty level, $F(6,372)=12.63$, $p<0.01$. MNE over the four difficulty levels was in order 2.46 (level 1), 2.34 (level 2),

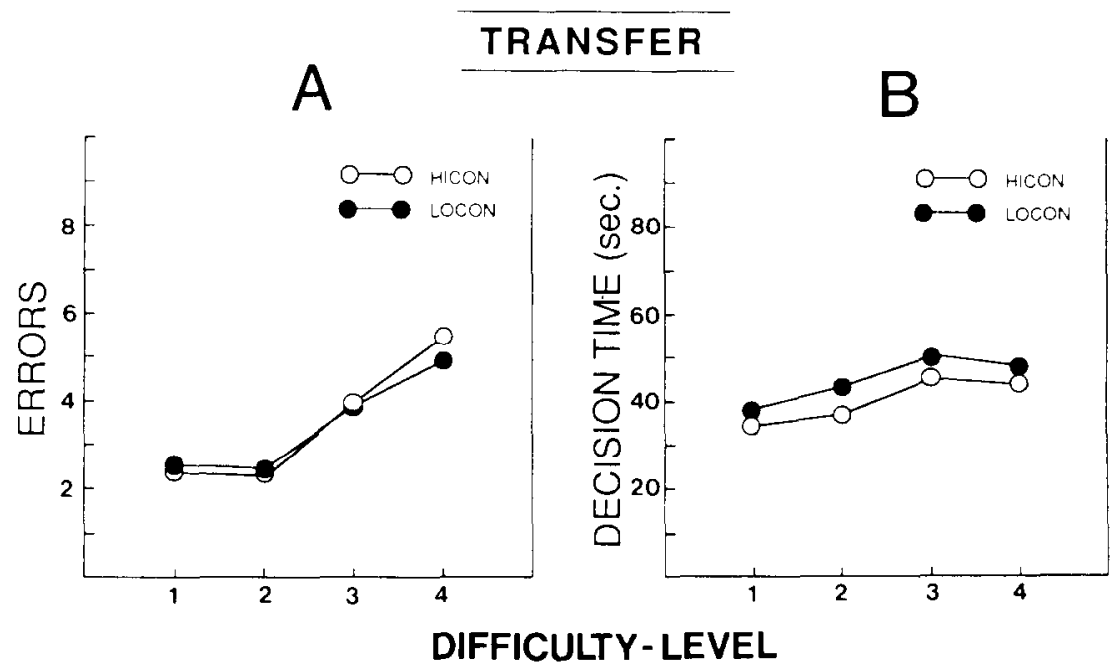

Fig. 4. The mean number of errors (4A) and the mean decision times (4B) on transfer tests of the high contextual interference group (HICON) and the low contextual interference group (LOCON) as a function of difficulty level. 
3.92 (level 3), and 4.93 (level 4). MDT over the four difficulty levels was $37.01 \mathrm{sec}$ (level 1), $39.61 \mathrm{sec}$ (level 2), $47.67 \mathrm{sec}$ (level 3), and $45.74 \mathrm{sec}$ (level 4), respectively. The ANOVAs on both error data, $F(3,186)=16.67, p<0.01$, and decision time data, $F(3,186)=14.52, p<0.01$, reached statistical significance as well. Thus, as predicted, the higher difficulty levels produced more errors and longer decision times than the lower difficulty levels. This result is again consistent with earlier findings that the provision of cues can reduce the difficulty level of the task (Lintern 1985).

Finally, the MANOVA indicated a significant effect for Testing moment, $F(2,61)$ $=5.66, p<0.01$. The subsequent ANOVA on decision time data reached statistical significance as well, $F(1,62)=4.92, p<0.05$, which indicated the expected performance decrement between the first $(\mathrm{MDT}=41.10 \mathrm{sec}$.) and second test $(\mathrm{MNE}=43.79$ sec.).

\section{General discussion}

The purpose of the present study was to determine the effect of contextual interference on the retention and transfer of procedures of different difficulty levels that have no procedural steps in common. In general, results showed that supplying a different number of cues during training was an effective operationalization producing different difficulty levels of the procedures to be learned. Furthermore, three major conclusions can be inferred from the results of our study.

First, as retention data clearly show, increasing task difficulty during training can facilitate learning. We suggest that this result can be accounted to an intensified use of controlled processes, that is to a delay in automatization of task performance. This finding could be of great practical importance. It suggests to instructional designers and trainers that immediate improvements in skill performance that can be expected if the learning situation is kept relatively simple, may be sacrificed if a maximum retention performance is the primary goal.

Second, the results of our study provided only minimal support (recall the acquisition data) for results of earlier studies on the contextual interference effect. So far, application of contextual interference in instructional settings only has been proven useful if the tasks to be learned are relatively easy. We contend that for those easy tasks, contextual interference raises the difficulty level in training. In our view, this procedure induces the use of controlled processes or delays automatization of task performance and hence can result in higher retention and transfer performances. This explanation is very similar to Lee and Magill's 'cognitive effort' explanation of contextual inter- 
ference effects. However, if tasks become more complicated, as in our study, the difficulty level of the task itself intensifies the use of controlled processing. There is no longer a need to artificially raise it by application of contextual interference. The extra effort induced by contextual interference is, as Shea and Zimny's (1983) 'elaboration' explanation suggests, applied to reveal relationships in the learning material. However, if the tasks have no elements in common, as the procedures in our study, the observed extra investment of controlled processing during training cannot be used to make abstractions from the learning material and thus is obviously more or less wasted. This would explain why the effect of contextual interference was not present in our study. Therefore, we would conclude that contextual interference may indeed intensify the use of controlled processing or delay automatization of task performance but is only effective for transfer if tasks are relatively easy or if relationships can be discovered in the learning material. Thus, we suggest that both Lee and Magill's 'cognitive effort' explanation and Shea and Zimny's 'elaboration' explanation should be integrated in order to adequately explain the contextual interference effects.

Third, since we did not find an interaction of condition with task difficulty, our study did not reveal whether training under conditions of high contextual interference has a negative effect on retention and transfer if the difficulty of a task crosses a certain level. For procedures that have no overlap, our results suggest that there exists at least a range in which practice under high and low contextual interference conditions results in identical retention and transfer performances. More research will be needed to reveal the exact relationship between the amount of contextual interference and task difficulty, for instance for even more complicated tasks or for tasks that do have elements in common.

\section{References}

Adams, J.A., 1987. Historical review and appraisal of research on the learning, retention, and transfer of human motor skills. Psychological Bulletin 101, 41-74.

Annett, J. and J. Piech, 1985. The retention for a skill following distributed training. Programmed Learning and Educational Technology 22, 182-185.

Annett, J. and J. Sparrow, 1985. Transfer of training: A review and practical implications. Programmed Learning and Educational Technology 22, 116-124. 
Battig, W.F., 1979. 'The flexibility of human memory'. In: L.S. Cermak and F.I.M. Craik (eds.), Levels of processing and human memory. Hillsdale, NJ: Erlbaum. pp. 23-44.

Del Rey, P., E.H. Wughalter and M. Whitehurst, 1982. The effects of contextual interference of females with experience in open sport skills. Research Quarterly for Exercise and Sport 53, $108-115$.

Eberts, R.E., 1987. Internal models, tracking strategies, and dual-task performance. Human Factors 29(4), 407-419.

Fisk, A.D. and W. Schneider, 1984. Memory as a function of attention, level of processing, and automation. Journal of Experimental Psychology: Learning, Memory, and Cognition 19 , $181-197$.

Jelsma, O. and J.M. Pieters, 1989. Practice schedule and cognitive style interaction in learning variants of a maze lask. Journal of Applied Cognitive Psycholoyy 3, 73-83.

Johnson, P., 1984. 'The acquisition of skill'. In: M.M. Smyth and A.M. Wing (eds.), The psychology of human movement. London: Academic Press. pp. 215-239.

Kieras, D. and P.G. Polson, 1985. An approach to the formal analysis of user complexity. International Journal of Man-Machine Studies 22, 365-394.

Lee, T.D. and R.A. Magill, 1983. The locus of contextual interference in motor skill acquisition. Journal of Experimental Psychology: Learning, Memory and Cognition 9, 730-746.

Lee, T.D. and R.A. Magill, 1985. 'Can forgetting facilitate skill acquisition?' In: D. Goodman, R.B. Wilburg and I.M. Franks (eds.), Differing perspectives in motor learning, memory and control. Amsterdam: North-Holland. pp. 3-23.

Lee, T.D., R.A. Magill and D.J. Weeks, 1985. Influence of practice schedule on testing schema theory predictions in adults. Journal of Motor Behavior 17, 283-299.

Lintern, G., 1985. 'Context and dependency effects in skill acquisition'. In: R.E. Eberts and C.G. Eberts (eds.), Trends in ergonomics/human factors II. Amsterdam: North-Holland. pp. $249-255$.

Magill, R.A. and T.D. Lee, 1984. Contextual interference effects in motor skill acquisition: Interference or elaboration benefit. Paper presented at the Olympic Scientific Congress, Eugene, OR, USA.

Romiszowski, A.J., 1984. Producing instructional systems. London: Kogan Page.

Salmoni, A.W., R.A. Schmidt and C.B. Walter, 1984. Knowledge of results and motor learning: A review and critical reappraisal. Psychological Bulletin 95(3), 355-386.

Schneider, W. and R.M. Shiffrin, 1977. Controlled and automatic human information processing: I. Detection, search, and attention. Psychological Review 84, 1-66.

Shea, J.B. and R.L. Morgan, 1979. Contextual interference effects on the acquisition, retention and transfer of a motor skill. Journal of Experimental Psychology: Human Learning and Memory 5, 179-187.

Shea, J.B. and S.T. Zimny, 1983. 'Contexts effects in memory and learning movement information'. In: R.A. Magill (ed.), Memory and control of action. Amsterdam: North-Holland. pp. $345-366$.

Shea, J.B. and S.T. Zimny, 1988. 'Knowledge incorporation in motor representation'. In: O.G. Meijer and K. Roth (eds.), Complex movement behaviour. Amsterdam: North-Holland. pp. 289-314.

Shiffrin, R.M. and W. Schneider, 1977. Controlled and automatic human information processing: II. Perceptual learning, automatic attending, and a general theory. Psychological Review 84 , $127-190$.

Simons, P.R.J. and G. Beukhof (eds.), 1987. Regulation of learning. The Hague: SVO. 